\title{
Primary Outcomes of a Healthy Lifestyle Intervention for Latino Families
}

\author{
Gail C. Frank $\circledast^{1,6}$, Selena T. Nguyen Rodriguez $\varpi^{2,6}$, Mara Bird $\varpi^{2,6}$, Melawhy Garcia $\circledast^{2,6}$, Natalia \\ Gatdula $^{2,6}$, Erika Centinaje ${ }^{3,6}$, Mayra Rascón ${ }^{4,6}$, and Britt Rios-Ellis $\oplus^{5,6}$ \\ ${ }^{1}$ Department of Family and Consumer Sciences, California State University Long Beach \\ ${ }^{2}$ Department of Health Science, California State University Long Beach \\ ${ }^{3}$ Health Education Department, The Permanente Medical Group, Inc., Santa Clara, CA \\ ${ }^{4}$ Department of Health Policy and Management, University of California Los Angeles \\ ${ }^{5}$ College of Health Sciences and Human Services, California State University Monterey Bay \\ ${ }^{6}$ Center for Latino Community Health, Evaluation and Leadership Training, California State University \\ Long Beach
}

\begin{abstract}
Background and Purpose: Latinos, especially children, are disproportionately affected by obesity. Sanos y Fuertes is a community-based, participatory research project that created a culturally resonant, bilingual, healthy lifestyle intervention for Latinos in California. Results of the program for families are presented. Methods: The sample consisted of 390 Latino families with at least one 2 to 8 -year-old child. Confidential, self-report, paper-and-pencil surveys and objective anthropometrics were assessed. Paired sample t-tests evaluated changes between baseline-to-follow-up assessments. Results: A significant decrease was observed in children's percent body fat, but not in adults. Improvements were found in adult knowledge, frequency of food label use and serving MyPlate portions. Adult frequency of breakfast, fruit, and vegetable intake increased, while frequency of sugary beverage intake decreased. Concomitant increases in parent-reported frequencies of children's intake of breakfast, fruits, and vegetables were observed along with a less frequent intake of sugary beverage. Conclusion: Sanos y Fuertes is a promising, short-term healthy lifestyle education program which may lower obesity risk among Latino families. Replication in a randomized controlled trial is needed to establish its effectiveness among diverse Latino populations.
\end{abstract}

(C) 2020 and CC-BY 4.0 licensed by the authors.

Keywords: culturally- and linguistically-relevant, obesity prevention, community health workers

\section{Introduction}

Being highly prevalent among Latino children, obesity increases risk for future health problems, such as diabetes (CDC, 2017; Hales et al., 2018; Ogden et al., 2018; Skinner et al., 2018). Obesity interventions for Latinos have had limited success (Branscum \& Sharma, 2011). Culturally- and linguistically-relevant health education including Hispanic/Latino (hereafter, Latino) values, e.g., familismo, is more likely to be successful promoting healthy lifestyle patterns in families because it integrates strategies and recommendations that are feasible and realistic within the context of cultural norms and practices (Rios-Ellis et al., 2014; Crespo et al., 2012).

Important corollaries for community-based practitioners is incorporating promotores de salud. Promotores are lay health workers who are generally regarded as leaders in their community, who community members are more likely to trust and heed their health advice (Hartzler et al., 2018). Integration of promotores into intervention development and delivery enhances access to the community, increases social and cultural relevance, and supports successful outcomes (Hilfinger Messias et al., 2013; Luque, Mason, Reyes-Garcia, Hinojosa, \& 
Meade, 2011; Philis-Tsimikas, Fortmann, LlevaOcana, Walker, \& Gallo, 2011).

The current study aimed to improve upon previous interventions by utilizing novel approaches and training practices. Integrating promotores (hereafter termed promotoras as all were female in Sanos y Fuertes) into the recruitment and engagement of participants as well as assisting with hands-on graduate student training is beneficial. It not only supports the graduate students' personal development but increases the community participants' belief and confidence in the program while increasing the potential for changing lifestyle behaviors. The role of graduate students who are culturally similar to the community is noteworthy as it provides a sustainable training model to address chronic health conditions. This paper describes the research design, results and family-based curriculum created to enhance both knowledge and skills for health promotion among Latino families around Long Beach, California.

\section{Methods}

\section{Study Design}

A lifestyle intervention, called Sanos y Fuertes (Healthy \& Strong), was delivered to Latino families (Frank et al., 2013) using a quasiexperimental design. While there was no control group, a pre-test-post-test design with follow-up was used, where participants' baseline measures were compared to outcomes assessed at 3-month follow-up (see Table 1 for additional details).

\section{Sample}

A total of 390 families were recruited using convenience sampling on a rolling basis across four years, with 328 completing all measurements at baseline and at 3-month follow-up, yielding an $84.1 \%$ completion rate. Participants were recruited by four promotoras at collaborating community sites: St. Mary's Medical Center, Spane Park Community Center, Fairfield Family YMCA and Centro Salud es Cultura. Eligibility criteria included selfidentifying as Latino, at least 18 years of age, having one child or grandchild (if grandparent lived in the home and was a primary caregiver) 2-8 years old, and residing in Los Angeles County.

\section{Procedures}

Consent and survey forms were completed in the participants' language of choice (predominantly Spanish). All measures were collected by promotoras and graduate research fellows (GRFs) who were trained according to standardized protocols. See Table 1. The same four promotoras recruited and implemented all retention activities, assisted with development of the curriculum, and taught the classes. Seven different Latino GRFs in health-related master's degrees (social work, nutrition, community health education) were selected annually for five consecutive years to obtain experiential learning in community-based participatory research. GRFs had a 1-year commitment of 20hours/week assisting with curriculum development, implementation and obtaining pre/post and 3-month follow-up assessments.

Baseline Data Collection. Participants attended a data collection session on a Thursday or Friday (6-9 p.m.) and the interactive educational session (charla) the following Saturday morning from 9 a.m.-2:00 p.m. including breakfast and lunch. During the data collection session, caregivers arrived with the 2 to 8 year-old-child (if parents had multiple children within the age range, the youngest child was the participant), and provided written informed consent. Details regarding measures are provided in Table 1. Parents then completed demographic and baseline questionnaires which assessed parent knowledge, self-efficacy (Fitzgerald, 2004), intentions, food label use (Fitzgerald, Damio, Segura-Pérez, \& Pérez-Escamilla, 2008), and MyPlate use; parent and child intake were measured by food frequency questionnaires (Fitzgerald et al., 2008) as well as a single item for breakfast (Bryant et al., 2008). Anthropometric measures were taken in light clothing without shoes or socks. The caregivers' height (stadiometer), weight and percent body fat (Tanita bioimpedance scale model SC-331S) and waist circumference (tape measure; 
triplicate measures) were assessed. Measured height and weight were obtained for all children, but percent body fat was only collected for children over 4 (the bioimpedance scale has not been validated to provide body composition of children under 5).

Table 1.

Baseline \& 3-Month Follow-up Measures

\begin{tabular}{|c|c|}
\hline \multicolumn{2}{|l|}{ Adults } \\
\hline Measure & Details \\
\hline Anthropometric & $\begin{array}{l}\text { CDC protocols for Height - Stadiometer } \\
\text { Weight } \% \text { Body Fat/BMI - Tanita Scale } \\
\text { Waist - Tape Measure }\end{array}$ \\
\hline Demographic Form & Age, marital status, household size (total occupants), education, number of years in U.S. \\
\hline Lifestyle & Food label use (5-item Fitzgerald et al., 2008) \\
\hline & $\begin{array}{l}\text { Portions using MyPlate amounts, Breakfast consumption frequency, and Grocery shopping } \\
\text { decisions. 4-item original scale created. Example: Using the MyPlate guide, how much (or } \\
\text { what percentage) of your plate should be fruits and vegetables? } \\
\text { Answers: One-fourth (25\%), half }(50 \%) \text {, three-fourths }(75 \%) \text {, and all (100\%). }\end{array}$ \\
\hline Knowledge & $\begin{array}{l}\text { 20-item original created. Example: A 2-8 year old child's recommended portion size for } \\
\text { cooked vegetables is equal to the size of } \overline{\text { ? }} \\
\text { Answers: An adult's hand, the mother's fist, the child's fist, and they should eat as much } \\
\text { vegetables as possible. }\end{array}$ \\
\hline Self-Efficacy & $\begin{array}{l}\text { 10-item from Fitzgerald et al. } 2004 . \\
\text { Example: How confident are you (0 to 10) to give your children correct portion sizes? }\end{array}$ \\
\hline Behavioral Intent & $\begin{array}{l}\text { 10-item original scale created. } \\
\text { Example: How sure are you (0 to 10) that you will do this? ...convince my child to drink } \\
\text { fewer sugar-sweetened beverages every day? }\end{array}$ \\
\hline Food Frequency & $\begin{array}{l}\text { 50-item from Fitzgerald et al. } 2008 \text { adapted to Latino foods. } \\
\text { Example: Think about last week; mark the answer that best describes the number of times } \\
\text { your child ate each of these fruits. Please focus on the eating habits of your 2-8 year old } \\
\text { child. Papaya. Never, } 1 \text { during the week, 2-6 during the week, } 7 \text { or more during the week. }\end{array}$ \\
\hline Children 2-8 yea & \\
\hline Measures & Details \\
\hline Anthropometric & Same as above \\
\hline Food Frequency & Same as above; parent responded about child \\
\hline
\end{tabular}

Note. Trained bilingual staff collected all measures for adults and children

Post and Follow-up Survey Data Collection. The morning of the charla, families were served breakfast and the charla began at 10 a.m. Following the education sessions, participants completed post-surveys. Three-months after the charla, families completed follow-up surveys and anthropometric data were measured for both adults and children (at community sites or in the home, based on participant choice and availability). Only baseline and follow-up were analyzed for the current paper to focus on behavioral and anthropometric outcomes. Families were given a $\$ 25$ gift card upon completion of each data collection step: the baseline, the charla with post survey, and the 3month follow-up. All procedures were approved by the Institutional Review Board of California State University, Long Beach. 
Intervention Framework. The culturally- and linguisticallyresponsive Sanos y Fuertes intervention was developed over a year-long process and primarily based on Bandura's Social Cognitive Theory which purports that personal, behavioral, and environmental factors dynamically interact to lead to actual behaviors (Bandura, 2004). The curriculum was created with the goal to increase knowledge of and skills for reinforcing healthy lifestyles among Latino families. Charla topics were selected based on an extensive review of evidence-based literature, national guidelines to reduce health disparities and chronic disease, and results of ten local focus groups ranging from school-aged Latino children to grandparents (Garcia et al., 2019).

\section{Previous Findings that Informed the} Intervention Curriculum. Focus group findings revealed cultural perceptions of weight, e.g., positive view of plump or overweight in small children, lack of nutrition-related knowledge, permissive parenting practices, and that low socio-economic status increased risk for obesity among Latino families. Adult participants discussed how economic status influenced their selections and if income was greater in the U.S. versus Mexico, for example, then the ease and availability of fast food fueled that choice frequently for adults and children. In contrast, participants stated that local organic produce was more affordable and available in the participants' home country. Parents voiced their use of food as a reward which was a challenge for them and seen as a weak, parenting skill. Qualitative analysis surfaced the need to include intergenerational family members to promote healthy lifestyle changes to facilitate proper nutrition when parents are away during work hours (Garcia et al., 2019).

Framework for the Curriculum. A resulting framework used for the curriculum defined separate, age-appropriate child lessons (in English and Spanish when needed for younger children) and adult instruction (in Spanish) using $6^{\text {th }}$ grade reading level, easy-to-understand content. Composition of the individual sessions reflected national directives, such as "Dietary
Guidelines for Americans" and age-specific serving sizes for "My Plate/Mi Plato" food groups. Healthy preparation of common Latino foods and convenient, no to low-cost physical activity behaviors for Latino families were incorporated as well as identifying healthier beverage options not only to reduce sugar intake, but to lessen parents using sweetened beverages as a reward for good child behavior.

Refinement of the Curriculum. As the topics developed, a sequential review session was conducted for each topic by the academic research team, all promotoras and the GRFs followed by editing and refinement. A vibrant, artistic Power Point template was developed with culturally resonant visual aids/photos and activity sheets, standard font size for all text and a consistent flow of each topic. The template required an initial introduction of the topic, health-related facts with reference citations, basic concepts about food and health, motivational interviewing (MI) techniques embedded in select sessions to increase discussion, self-efficacy and intentions with open-ended questions, and a brief question and answer period followed by a complementary skill-development activity. Examples of a skilldevelopment activities include identifying the variation in number of servings of different food groups for family members based on label information, using beans, rice and oatmeal, demonstrating how to read and interpret different sections of the food label, comparing food labels to determine foods with a high salt, sugar and unhealthy fats content, selecting healthier options from menus when eating out, and learning how to model their own healthy behaviors to their children. These hands-on activities supported behavioral skill-building and included in-depth meal planning using a culturally relevant grocery shopping guide.

PowerPoint text for adult classes was written and visible for participants for each slide, i.e., left half of the slide written in Spanish and right half of the slide in English. Promotoras and GRFs were assigned to specific topics and had 
repetitive practice to assure ease with and a timely, standardized delivery of the content.

The resulting 4-hour interactive curriculum (see Table 2 and Table 3) was created as a realistic foundation for a healthy lifestyle with eating to promote both individual members' nutritional needs and family-oriented physical activity. It was delivered by the same four promotoras across all years and each cohort of bilingual, often bicultural, GRFs. PowerPoint presentations for each of the eight sessions were printed and placed in a binder with instructive information to guide the promotoras and GRFs teaching the classes. All materials were ultimately compiled into an attractive toolkit for future application and testing.

Table 2.

Charla Content for Children

\begin{tabular}{|c|c|}
\hline Content & Duration \\
\hline \multicolumn{2}{|l|}{ Birth to 2 years* } \\
\hline $\begin{array}{ll}\text { - } & \text { Infant/Childcare } \\
\text { - } & \text { Safe, soft toys } \\
\text { - } & \text { Crawling and walking } \\
\end{array}$ & All day \\
\hline \multicolumn{2}{|l|}{3 to 5 years** } \\
\hline $\begin{array}{l}\text { - The Very Hungry Caterpillar } \\
\text { Story } \\
\text { - "Eating the Alphabet: Fruits \& } \\
\text { Vegetables from A to Z" } \\
\text { - Pineapple Piñata }\end{array}$ & $\begin{array}{l}\text { 4-hours } \\
\text { - Arts and } \\
\text { crafts } \\
\text { - Structured } \\
\text { play }\end{array}$ \\
\hline \multicolumn{2}{|l|}{6 to 8 years** } \\
\hline $\begin{array}{l}\text { - Jack \& the Hungry Giant Story: } \\
\text { Eat Right with MyPlate; 'Keep } \\
\text { Your Body Healthy' drawing } \\
\text { - } \quad \text { Rainbow game using } 5 \text { senses }\end{array}$ & $\begin{array}{l}\text { 4-hours } \\
\text { - Arts and } \\
\text { crafts } \\
\text { - Structured } \\
\text { play }\end{array}$ \\
\hline
\end{tabular}

Note. Taught by: *GRFs and promotoras; ${ }^{* *} \mathrm{GRFs}$

Provision of Amenities. Given the contextual needs and assets among Latino families, facilitators for family participation included providing a healthy breakfast, lunch and snacks reinforcing not only good parenting (familismo), but also optimal nutrition. Additional culturally responsive facets included childcare for infants, age-relevant classes for children in the room adjacent to the parents with children's' freedom to walk to their parents, free parking and having the education location close to bus lines.

Analysis - Repeated-measures t-tests assessed changes from baseline to follow-up in knowledge, self-efficacy, intentions, food label use, MyPlate use, frequency of breakfast, fruit, vegetable and sugary beverage intake, percent body fat, waist circumference, and BMI. Statistical significance was set at $p<.05$, and Cohen's d-values provided estimates of effect size. All analyses were conducted using SPSS v. 23 (IBM Corp, Armonk, NY).

\section{Results}

The sample $(99.0 \%$ female) was primarily foreign-born $(92.3 \%)$ with years in the U.S. ranging from weeks to 45 years $(\mathrm{M}=15.00 \pm 1.10)$. The majority were married or living with a partner $(85.0 \%)$. Nearly two-thirds $(66.2 \%)$ had less than a high school diploma/GED and $95.7 \%$ of families were living in a severely crowded home. Average age for adults was $35.80 \pm 6.66$ years, with $29.6 \%$ overweight and $53.4 \%$ obese; children's mean age was $4.72 \pm 1.95$, with $15.6 \%$ overweight, $30.1 \%$ obese weight.

Statistical results for changes from baseline to follow-up are in Table 4. Adult knowledge, selfefficacy and intentions significantly improved. Frequency of food label use and MyPlate use also increased. Parents increased frequency of breakfast, fruit intake, vegetable intake, and decreased sugary beverage intake. Parentreported children's breakfast, fruit and vegetable frequency was higher given a lower sugary beverage intake. There were no changes in adult percent body fat, BMI, or waist circumference. Children's percent body fat significantly improved, but no change was found in BMI percentile. 
Table 3.

Content

Charla Content for Caregiver (Parent/Grandparent)

Importance of breakfast*

Duration \& Details

- Culturally relevant healthy breakfast

15 minutes

- Benefits of breakfast foods

- Whole wheat quesadilla with vegetables

- Motivational tips

- Alertness, attentiveness and concentration

- Prepare ingredients the night before

- Allow kids to help

- Role model

MyPlate** 40 Minutes

- Interactive discussion on MyPlate \& meal guide

- MI techniques to increase participants' self-efficacy using MyPlate guidelines

Portion sizes**

15 minutes

- Demonstrate measuring \& weighing

- Measuring cups, spoons, gram scale

- Using hand as a guide

- Examples of culturally relevant healthy meal plans

with appropriate portions sizes for adults and children

Nutrition Label \& Activities** 30 minutes

- Nutrition Label Activity

- Discussion of calories, portions, \% Daily Value

- Labels and Portions Activity

Sweetened Beverages**

- Measure beans and oatmeal

- Nutrition label activity: sugar content of drinks

- Answer questions about label information

- Discussion of recommended limit of sugar intake for men, women, and children

Grocery Shopping \& Activity**

20 minutes

- Identify healthier beverage

- Self-efficacy scale to measure participants' confidence limiting sweetened beverages

- Interactive discussion of current habits

- Presentation: "Ready, Set and Go!"

- Set shopping rules

- Self-efficacy scale

- Involve children in games with mystery vegetable

- Grocery Shopping Activity

40 minutes

- Tips to be an efficient shopper

- Planning, locating and purchasing healthier foods and beverages

- Assess participants' confidence planning when grocery shopping

- Guidance when shopping with children

- Choose 3 healthy options in each: dairy, poultry, fruits, vegetables, cooking oils

Physical Activity Action**

15 minutes

- Adults and children led in group exercise

- Warm-up; proper stretching techniques

- Exercise circuit; Resistance training

Sedentary behavior**

15 minutes

- Recommendations for screen time (ST)

- Self-efficacy

- Tips for parental involvement with children

- Measure confidence to limit own and children's ST, $\&$ increase physical activity

Summary of Charla Topics***

25 minutes

- Reviewed charla sessions and topics

- Key points/summary

- Post-test

- Collect post survey data

Note. Taught by: *GRFs and promotoras; **GRFs; **Taught by promotoras 
Table 4.

Changes from Baseline to Follow-Up

\begin{tabular}{|c|c|c|c|c|c|}
\hline & \multicolumn{2}{|c|}{$\mathrm{M}(\mathrm{SD})$} & \multirow[b]{2}{*}{$\mathrm{t}$} & \multirow[b]{2}{*}{$\mathrm{p}$} & \multirow{2}{*}{ d } \\
\hline & Baseline & Follow-up & & & \\
\hline \multicolumn{6}{|l|}{ Caregiver } \\
\hline Knowledge & $11.56(.17)$ & $13.68(.16)$ & -13.87 & $<.001$ & .761 \\
\hline Self-efficacy & $7.29(.10)$ & $8.63(.07)$ & -12.95 & $<.001$ & .733 \\
\hline Intentions & $8.01(.10)$ & $8.92(.07)$ & -9.79 & $<.001$ & .558 \\
\hline Food label use & $1.24(.04)$ & $1.83(.03)$ & -14.89 & $<.001$ & $.828)$ \\
\hline MyPlate use & $0.70(.05)$ & $1.84(.04)$ & -21.93 & $<.001$ & 1.223 \\
\hline Breakfast* & $5.94(.10)$ & $6.35(.07)$ & -4.70 & $<.001$ & .268 \\
\hline Fruit* & $2.10(.02)$ & $2.15(.02)$ & -4.07 & $<.001$ & .223 \\
\hline Vegetable* & $2.18(.02)$ & $2.26(.02)$ & -4.67 & $<.001$ & .256 \\
\hline Sugar-sweetened beverage* & $1.97(.02)$ & $1.89(.02)$ & 2.92 & .004 & .167 \\
\hline BMI & $30.81(6.55)$ & $30.65(6.20)$ & 1.03 & .337 & .024 \\
\hline Percent body fat & $37.09(7.46)$ & $37.23(7.79)$ & -0.96 & .337 & .018 \\
\hline Waist circumference & $97.73(12.68)$ & $97.51(12.45)$ & 0.67 & .505 & .017 \\
\hline \multicolumn{6}{|l|}{ Child } \\
\hline Breakfast* & $6.45(.07)$ & $6.74(.04)$ & -13.87 & $<.001$ & .761 \\
\hline Fruit* & $2.08(.02)$ & $2.15(.02)$ & -3.98 & $<.001$ & .232 \\
\hline Vegetable* & $1.88(.02)$ & $2.01(.02)$ & -3.49 & .001 & .192 \\
\hline Sugar-sweetened beverage* & $1.92(.02)$ & $1.87(.02)$ & 2.13 & .034 & .117 \\
\hline BMI percentile & $67.01(30.66)$ & $66.59(31.51)$ & 0.38 & .702 & .013 \\
\hline Percent body fat & $26.66(9.29)$ & $25.33(9.13)$ & 2.59 & .011 & .221 \\
\hline
\end{tabular}

*Frequency of behavior was assessed

\section{Discussion}

Maintaining a healthy lifestyle and being able to implement healthy behaviors is essential if Latino families are expected to practice obesity risk-reducing eating and exercise behaviors. These may have been practiced in their native country but have since changed given the eating and activity choices available to them in the U.S. Since most adults were immigrants, attention should be given to ensuring that Latinos have access to healthy and familiar food selections within their U.S. communities. Insights from focus groups representing different ages of children, adults and older adults (Garcia et al., 2019), were integrated into a cultural- and linguistically relevant curriculum based on national recommendations for nutritional goals and serving sizes of foods and beverages.

Pre-to-3-month-follow-up measurement noted improvements in frequency of adult and parentreported children's breakfast, fruit, vegetables and sugary beverage frequency. A significant decrease in children's, but not adults', percent body fat was observed. Although no significant decrease was noted in parents' adiposity estimates, their weights remained stable. Given the large sample size, effect sizes increase confidence that beyond statistical significance most of the changes found were practically meaningful; with the exception of frequency of sugary beverage, child fruit and vegetable intakes, all changes were found to have at least a small effect size. The lower magnitude of dietary change is not a surprise, given the brevity of the intervention; providing additional behavioral intervention strategies across a longer period of time is more likely to result in greater positive improvements in dietary intake.

The results of this study are consistent with the findings of a longer 10-week community-based program aiming to increase fruit and vegetable consumption among child-parent dyads (JarpeRatner, Folkens, Sharma, Daro \& Edens, 2016). Similar to other studies, our study showed no significant decreases in child's BMI although positive changes were observed in nutritional intake (Crespo et al., 2012). The reported 
changes noted in our educational intervention may be the initial steps that are essential if a pathway for improving overall lifestyle and BMI among Latino families are to occur. With continued commitment to healthier food and physical activity choices leading to changes in lifestyle, adiposity decline may be seen. In theory, positive, sustained behaviors outside a research model must be internalized and families must believe that the changes they make will result in improved health. Further, if families can support each other, which is not foreign to the beliefs of the Latino culture, then success in creating a new lifestyle could occur.

High participation in this educational intervention and assessments may be attributed to the positive, supportive and caring environment created by the promotoras at all contact points. This highlights the effectiveness of implementing culturally-relevant practices which involved tailoring demonstrations during sessions with healthier forms of preferred Latino foods and giving environmental support. This included personal needs like free childcare for siblings of the index child, serving tasty and healthy snacks and lunch and providing an appropriate economic incentive to honor participants' time. Concomitant classes for the 3 to 5 and 5 to 8 -year-old children, who were taught the same topics, reinforced the information given to parents/caregivers.

\section{Limitations}

The health education session, although interactive to include hands-on learning, was brief and could have resulted in larger changes if it had been extended or contained 'booster' reinforcing sessions possible with increased funding for intervention. An interim focus group to seek information about the new challenges faced as the families tried healthier lifestyle changes would have been instructive for the staff.

The lack of a control group prevents the research team from attributing the changes found only to the charla. Further, the food frequency questionnaire had a limited range of responses and did not capture actual food intake, thus we are unable to assess if amount of fruit and vegetable intake improved. Some measures were adapted from the original and some were developed for the project; while we did not undertake standard psychometric testing of these measures, they were evaluated by promotoras for clarity and face validity and revised accordingly; quality of measures should be tested in future research. Given the self-report nature of data, responses may have been affected by recall bias and social desirability. A longer interval between intervention and follow-up assessment would have allowed for evaluation of whether changes were sustained. Finally, the sample was primarily comprised of Mexican $(86.5 \%)$ immigrants, thus findings may not generalize to other Latino heritage groups or those that were born in the U.S. Despite the research limitations, the findings indicate that health education lifestyle interventions using multiple culturally-congruent approaches with quality controls may improve the well-being of a primarily Mexican immigrant population at high risk for chronic illness living in low-income areas.

\section{Conclusion}

Family-based, lifestyle interventions can create short-term attitudinal, behavioral and anthropometric effects when sensitive to the culture of the participants and implemented in partnership with the target population. The program described herein was culturallyresonant employing promotoras who worked closely and consistently embellishing familismo and strengthening the participants' well-being. Latino parents and children were receptive as they believed the program was sensitive to their needs. The observed decline in children's percent body fat is noteworthy indicating that improving a child's and parent's nutrition and physical activity knowledge, intentions and behavior may influence a child's health by lowering risk factors for chronic disease.

Sanos y Fuertes can serve as a positive blueprint for future programs to benefit Latino communities and Latino-serving practitioners. 
Healthy lifestyle intervention may result in statistically significant reported behavior change. The model demands a practical design, dedicated staff, and a trusting, caring relationship between staff, students and the community participants. When graduate students have experiential learning in which they train alongside and by community health workers, they can refine their skills not only for the immediate implementation but also for their future practice in communities. Participants, who trust individuals who teach them important skills, may increase their knowledge and become stronger leaders within their homes and communities. This can foster broader environmental changes to reduce health disparities, lessen their personal and their family's chronic disease risk, such as for obesity, and improve their overall quality of life.

\section{References}

Bandura, A. (2004). Health promotion by social cognitive means. Health Education and Behavior, 31(2), 143-164.

Bryant, M. J., Ward, D. S., Hales, D., Vaughn, A., Tabak, R. G., \& Stevens, J. (2008). Reliability and validity of the Healthy Home Survey: A tool to measure factors within homes hypothesized to relate to overweight in children. International Journal of Behavioral Nutrition and Physical Activity, 5(1), 23. doi:10.10.1186/1479-5868-5-23

Branscum, P. \& Sharma, M. (2011). A systematic analysis of childhood obesity prevention interventions targeting Hispanic children: Lessons learned from the previous decade. Obesity Reviews, 12, e151-e158.

Centers for Disease Control. (2017). Health United States, 2016: With chartbook on long-term trends in health (p. 265). [PDF File]. Hyattsville, MD. Retrieved from http://www.cdc.gov/nchs/data/hus/hus16.pdf

Crespo, N., Elder, J.P., Ayala, G.X, Slymen, D.J., Campbell, N.R., Sallis, J.F., McKenzie, T.L., Baquero, B., \& Arredondo, E.M. (2012). Results of a multi-level intervention to prevent and control childhood obesity among Latino children: The Aventuras para Niño's Study, Annals of Behavioral Medicine, 43(1), 84-100.

Hilfinger Messias, D. K., Parra-Medina, D., Sharpe, P. A., Treviño, L., Koskan, A. M., \& MoralesCampos, D. (2013). Promotoras de salud: Roles, responsibilities, and contributions in a multi-site community-based randomized controlled trial. Hispanic Health Care International, 11, 62-71.

Fitzgerald, N. (2004). Identification of risk factors mediating the associations of socioeconomic, cultural, and demographic factors with type 2 diabetes across ethnic groups. Dissertations Collection for University of Connecticut, Paper AAI3165990, from http://digitalcommons.uconn.edu/dissertations/AAI3165990.

Fitzgerald, N., Damio, G., Segura-Pérez, S., \& Pérez-Escamilla, R. (2008). Nutrition knowledge, food label use, and food intake patterns among Latinas with and without type 2 diabetes. Journal of the American Dietetic Association, 108(6), 960-967.

Frank, G.C., Beaudoin, J., Rascón, M., \& Rios-Ellis B. (2013). Development of a culturally relevant nutrition promotion course for Latinos. Journal of the American Association of Family and Consumer Sciences, 105(1), 10-17.

Garcia, M.L., Gatdula, N., Bonilla, E., Frank, G.C., Bird, M., Rascón, M., \& Rios-Ellis, B. (2019). Engaging intergenerational Hispanic/Latinos to examine factors influencing childhood obesity using the PRECEDE-PROCEED model. Maternal and Child Health Journal, 23, 802-810. doi:10.1007/s10995-018-02696-y

Hales, C.M., Fryar, C.D., Carroll, M.D., Freedman, D.S., \& Ogden, C.L. (2018). Trends in obesity and severe obesity prevalence in US youth and adults by sex and age, 2007-2008 to 20152016. Journal of the American Medical Association, 319(16), 1723-1725. doi:10.1001/jama.2018.3060 
Hartzler, A. L., Tuzzio, L., Hsu, C., \& Wagner, E. H. (2018). Roles and functions of community health workers in primary care. Annals of Family Medicine, 16(3), 240-245. https://doi.org/10.1370/afm.2208

IBM Corp. Released 2015. IBM SPSS Statistics for Windows, Version 23.0. Armonk, NY: IBM Corp. Jarpe-Ratner, E., Folkens, S., Sharma, S., Daro, D., \& Edens, N.K. (2016). An experiential cooking and nutrition education program increases cooking self-efficacy and vegetable consumption in children in grades 3-8. Journal of Nutrition Education and Behavior, 48(10), 697-705, https://doi.org/10.1016/j.jneb.2016.07.021

Luque, J. S., Mason, M., Reyes-Garcia, C., Hinojosa, A., \& Meade, C. D. (2011). Salud es Vida: Development of a cervical cancer education curriculum for promotora outreach with Latina farmworkers in rural southern Georgia. American Journal of Public Health, 101, 2233-2235.

Ogden C.L., Fryar, C.D., Hales, C.M., Carroll, M.D., Aoki, Y., Freedman, D.S. (2018). Differences in obesity prevalence by demographics and urbanization in US children and adolescents, 2013-2016. Journal of the American Medical Association, 319(23), 2410-2418. doi:10.1001/jama.2018.5158

Philis-Tsimikas, A., Fortmann, A., Lleva-Ocana, L., Walker, C., \& Gallo, L. C. (2011). Peer-led diabetes education programs in high-risk Mexican Americans improve glycemic control compared with standard approaches: a project dulce promotora randomized trial. Diabetes Care, 34, 1926-1931.

Rios-Ellis, B., Garcia-Vega, M., Frank, G.C., Gatdula, N. \& Galvez G. (2014). Comienzo Sano, Familia Saludable: Addressing Latino childhood obesity through a community-based participatory research and bilingual family- focused curriculum in underserved communities. Johns Hopkins University Press, 255-262.

Skinner, A.C., Ravanbakht, S.N., Skelton, J.A., Perrin, M., and Armstrong, S. C. (2018). Prevalence of obesity and severe obesity in US children, 1999-2016. Pediatrics, 141(3), e20173459. doi: 10.1002/oby.21497

U.S. Department of Health and Human Services and U.S. Department of Agriculture (2015). 2015-2020 Dietary Guidelines for Americans. 8th ed. Retrieved from http://health.gov/dietaryguidelines/2015/

\section{Acknowledgements}

This study was supported by the United States Department of Agriculture, grant number 2011-6700230152. The research and implementation team at CSULB worked closely with and is most appreciative of the tremendous contributions of the team of promotoras of the Center for Latino Community Health, Evaluation and Leadership Training, the Center project staff and graduate students, the Sanos y Fuertes community partners, and the participant families who made this project possible.

Corresponding Author Information

Gail C. Frank (ORCID ID: 0000-0002-1853-0371)

Professor, Department of Family and Consumer Sciences

California State University, Long Beach

Long Beach, CA 90840-0501

Tel: (562) 985-4494

Email: Gail.Frank@csulb.edu 